\title{
Genotype-Phenotype Correlation and Therapeutic Rationale in Hyperkalemic Periodic Paralysis
}

\author{
Karin Jurkat-Rott and Frank Lehmann-Horn \\ Department of Applied Physiology, Ulm University, Ulm, Germany
}

\begin{abstract}
Summary: Familial hyperkalemic periodic paralysis (PP) is a dominantly inherited muscle disease characterized by attacks of flaccid weakness and intermittent myotonia. Some patients experience muscle stiffness that is aggravated by cold and exercise, bordering on the diagnosis of paramyotonia congenita. Hyperkalemic PP and paramyotonia congenita are allelic diseases caused by gain-of-function mutations of the skeletal muscle sodium channel, Nav1.4, which is essential for the generation of skeletal muscle action potentials. In this review, the functional and clinical consequences of the mutations and therapeutic strategies are reported and the differential diagnoses
\end{abstract}

discussed. Also, the question is addressed of whether hyperkalemic PP is truly a different entity than normokalemic PP. Additionally, the differential diagnosis of Andersen-Tawil syndrome in which hyperkalemic PP attacks may occur will be briefly introduced. Last, because hyperkalemic PP has been described to be associated with an $\mathrm{R} 83 \mathrm{H}$ mutation of a MiRP2 potassium channel subunit, evidence refuting disease-causality in this case will be discussed. Key Words: Hyperkalemic periodic paralysis, myotonia, hypokalemic periodic paralysis, Andersen-Tawil syndrome, voltage-gated sodium channel, channelopathies.

\section{INTRODUCTION}

Hyperkalemic periodic paralysis (PP), paramyotonia congenita (PC), and normokalemic PP are allelic muscle sodium channelopathies that include both hyper- and hypoexcitability of muscle fibers, frequently in the same patient at different times. Fortunately for the patients, the presenting symptoms of muscle stiffness (myotonia) and muscle weakness (paralysis) are intermittent. The attacks are typically triggered by exposure to cold (PC), ingestion of potassium-rich food (hyperkalemic PP), or rest after a heavy work load (hyperkalemic PP and normokalemic PP). The symptoms usually disappear spontaneously within hours or days, and attacks can be avoided by prophylactic medication and appropriate lifestyle adjustments. Nonetheless, symptoms can drastically interfere with the patient's ability to perform activities of daily life. In PP, progressive permanent weakness can make patients wheelchair-bound.

The present review concentrates on hyperkalemic PP, a disease that usually is associated with hyperkalemia

Address correspondence and reprint requests to: Frank LehmannHorn, Department of Applied Physiology, Ulm University, AlbertEinstein-Allee 11, 89081 Ulm, Germany.E-mail: frank.lehmann-horn@ uni-ulm.de. during an attack of weakness, and on the clinically related PC. In-depth information may be found in a recent book chapter. ${ }^{1}$

\section{HYPERKALEMIC PP: CLINICAL FEATURES}

In hyperkalemic PP, potassium-rich food or rest after exercise may trigger an attack of flaccid limb weakness, or, less frequently, weakness of the muscles of the eyes, throat, and trunk. Additional triggers or modifiers include a cold environment, emotional stress, fasting, and pregnancy. Typically, a spontaneous attack commonly starts in the morning before breakfast, lasts up to an hour, and then disappears. The muscle stretch reflexes are $a b-$ normally diminished or absent during the period of paralysis. At the end of an attack of weakness, elimination of potassium via the kidney and reuptake of potassium by the muscle can cause transient hypokalemia, which can lead to the misdiagnosis of hypokalemic PP. Sustained mild exercise after a period of strenuous exercise may postpone or prevent weakness in the exercising muscle groups and improve the recovery of muscle force while resting muscles become weak. ${ }^{2}$

The attacks of flaccid muscle weakness associated with an increase in serum potassium usually begin in the first decade of life. Adult onset points to other diagnoses, 
such as the Andersen-Tawil syndrome or secondary acquired forms of hyperkalemic PP. In typical hyperkalemic PP, the attacks then increase in frequency and severity during puberty, adolescence, and early adulthood. During adulthood, attacks do not much vary until about the age of 40 years, after which the frequency of attacks declines considerably. Depending on the underlying mutation, some older individuals develop a chronic progressive myopathy ${ }^{3}$ with permanent weakness that may go unrecognized by the patient and the physician. The slowly progressive muscle degeneration affects mainly the pelvic girdle and proximal and distal lower limb muscles (FIG. 1).

Between attacks, serum potassium and sodium as well as muscle strength are usually normal. Serum creatine kinase concentration is elevated, sometimes 5 to 10 times above the normal range. Typically, hyperkalemic PP is associated with a myotonia that is mild and does not impede voluntary movements. Myotonia (i.e., increased membrane excitability) is most readily observed in the facial, lingual, thenar, and finger extensor muscles and, if present in the EMG, it supports the diagnosis of hyperkalemic PP in contrast to all other forms of PP. In some patients, these muscles-and particularly the calf muscles-are hypertrophic. The myotonia increases at the beginning of an attack and is absent during a fullblown attack of weakness. The myotonia is not present in all patients, and indeed is absent in almost half of the patients harboring T704M (the most frequent mutation for the disorder). ${ }^{4}$ In some individuals, paresthesias, probably induced by the hyperkalemia, herald the weakness. Cardiac arrest due to hyperkalemia-induced arrhythmia rarely occurs during an attack..$^{5,6,7}$

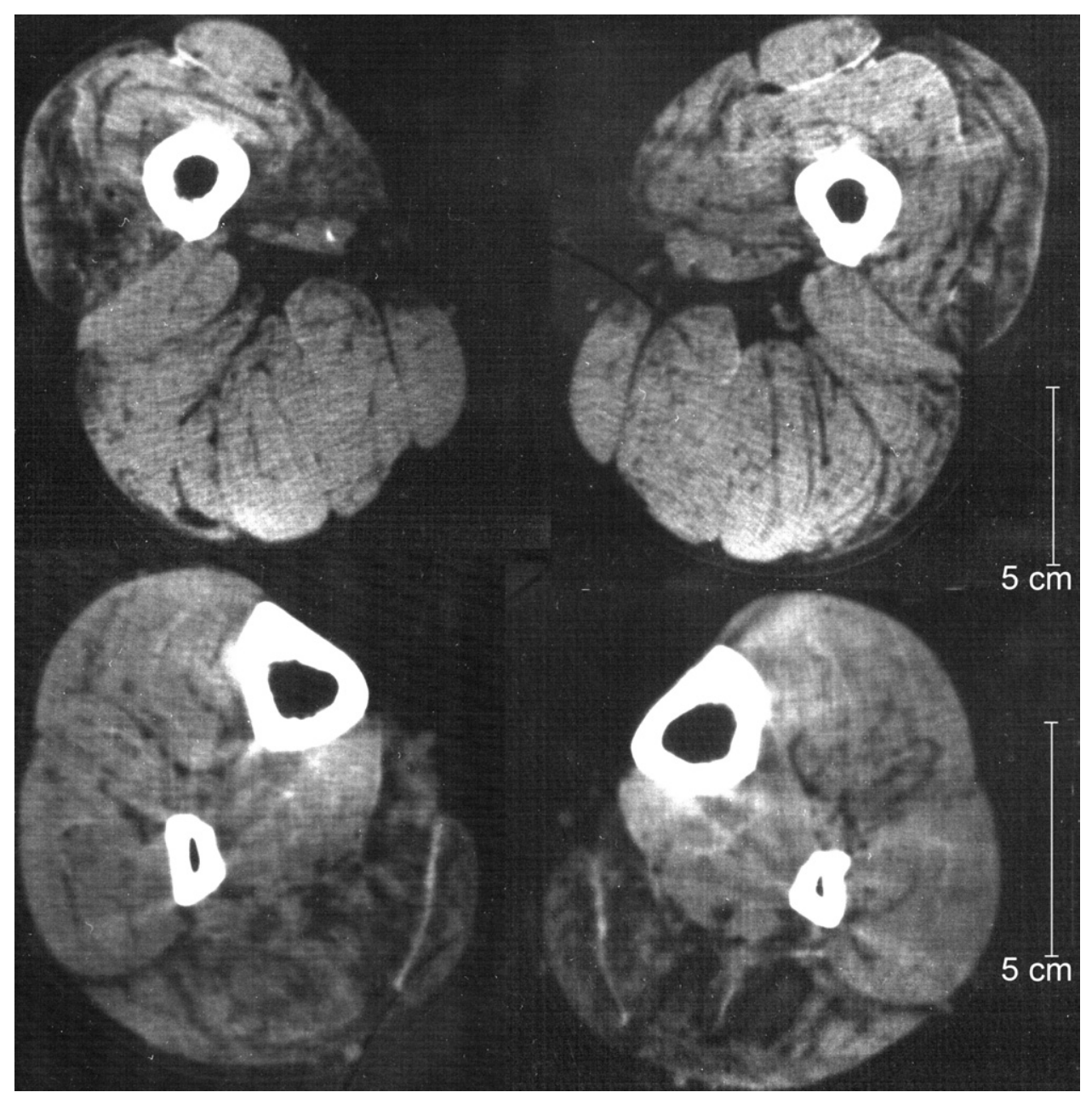

FIG. 1. Computed tomography scan from 1987 of the upper (top) and lower legs (bottom) of a male hyperkalemic periodic paralysis (PP) patient at age of 41 years who harbors the T704M mutation and developed persistent slight weakness at 30 years of age. Note the bilateral degeneration of the triceps surae muscles and the less pronounced degeneration of the upper leg muscles. In addition, the patient suffers from paralytic attacks that last up to $2 \mathrm{~h}$ and are provoked by exercise and preceded by myotonic stiffness. As of writing he was still ambulatory but unable to climb stairs or to walk even slightly ascendant streets. A recent MRI scan (not shown) demonstrated a progression of the fatty muscle degeneration that also affected both tibialis anterior muscles and led to muscle atrophy. His father, who was affected by frequent severe attacks of generalized weakness, also had muscle wasting and was confined to a wheelchair from the age of 54 years. 
TABLE 1. Genotype-Phenotype Correlations of Hyperkalemic PP Mutations

\begin{tabular}{lrll}
\hline Mutation & RF, $\%^{8}$ & \multicolumn{1}{c}{ Special Features } & First Report \\
\hline L689I & $<1$ & Pain due to muscle cramping & Bendahhou et al., 2002 $^{17}$ \\
I693T & $\sim 8$ & Cold-induced weakness* & Plassart et al., 1996 $^{18}$ \\
T704M & $\sim 30$ & Permanent weakness, myopathy & Ptacek et al., 1991 $^{19}$ \\
A1156T & $<1$ & Reduced penetrance & McClatchey et al., 1992 $^{9}$ \\
M1360V & $<1$ & Reduced penetrance & Wagner et al., 1997 $^{10}$ \\
M1370V & $<1$ & Paramyotomia in one family member, PP in others & Okuda et al., 2001 $^{20}$ \\
I1495F & $<1$ & Cramping pain, muscle atrophy & Bendahhou et al., 1999 $^{21}$ \\
M1592V & $\sim 13$ & Classic clinical features with EMG myotonia & Rojas et al., 1991 $^{16}$ \\
F1490L + M1493I & $<1$ & Malignant hyperthermia susceptibility & Bendahhou et al., 2000 $^{22}$ \\
\hline
\end{tabular}

$\mathrm{RF}=$ relative frequency among patients.

* Reported as paramyotonia congenita mutation.

${ }^{\dagger}$ The anesthesia-related events may have been exaggerated myotonic reactions, as in several other individuals with gain-of-function sodium channel mutations. ${ }^{38}$

Although substantiated data are not available, the prevalence of hyperkalemic PP is approximately $1: 200,000$ and the penetrance is high $(>90 \%){ }^{8}$ Only a few individuals with rare mutations are clinically asymptomatic even though they are obligate carriers or show myotonic bursts on EMG. ${ }^{9,10}$ De novo mutations have not yet been reported.

\section{NORMOKALEMIC PERIODIC PARALYSIS: A SEPARATE ENTITY?}

The term normokalemic PP was originally given to a variant described in the 1960s. The disorder resembled hyperkalemic PP in many aspects; the only real differences were the lack of increase in the concentration of serum potassium even during serious attacks, and the lack of a beneficial effect of glucose administration. ${ }^{11,12}$ The existence of normokalemic PP as a nosologic entity was questioned, because of the potassium sensitivity of the patients and the identification of the most frequent hyperkalemic PP mutations T704M or M1592V in such families, including the original family. ${ }^{4,13}$

Recently, a potassium-sensitive type of PP with normokalemia and episodes of weakness reminiscent of those in both hyperkalemic and hypokalemic PP was reported. This phenotype, also named normokalemic PP, is caused by $S C N 4 A$ mutations at codon $675 .{ }^{14}$ Codon 675 encodes an arginine in the voltage sensor of domain 2 of the sodium channel next to substitutions of R672 and R699 that cause hypokalemic PP type 2. Future studies will show whether normokalemic PP is a separate clinical entity and whether the normokalemia during an attack and the combination of features typical for hyperkalemic and hypokalemic PP can be used to determine the most probable localization of the causative mutation in SCN4A.

\section{HYPERKALEMIC PP: A GAIN-OF-FUNCTION SODIUM CHANNELOPATHY}

The disease is caused by missense mutations in SCN4A on chromosome region $17 \mathrm{q} 23.1 \sim \mathrm{q} 25.3$ encoding the voltage-gated sodium channel $\alpha$-subunit, $\mathrm{Na}_{\mathrm{v}} 1.4{ }^{15,16}$ The mutations and their relative frequencies among the patients are listed in Table $1 .^{9,10,16-22}$ Most $\mathrm{Na}_{\mathrm{v}} 1.4 \mathrm{mu}-$ tations are situated at inner parts of the transmembrane segments or in intracellular protein loops and affect structures that form the three-dimensional docking site for the fast inactivation particle (FIG. 2); any malformation may reduce the affinity between the "latch bar and the catch." The mutant channels avoid the fast inactivated state and, in contrast to normal $\mathrm{Na}^{+}$channels, reopen or flicker between the inactivated and the open state $^{23}$ (FIG. 3), corresponding to a gain-of-function defect. $^{10,21}$ As a result, sodium influx is increased, as shown in vitro ${ }^{24}$ and in vivo ${ }^{25}$ (FIGS. 4 and 5). This inward current is associated with a sustained membrane depolarization that increases the electrical driving force for potassium, and the resulting release of potassium from muscle elevates the serum potassium level. Sodium influx into muscle is accompanied by entrance of water into the fibers, causing higher serum concentrations, including a further increase in serum potassium. This is a vicious cycle, which spreads out and affects the surrounding muscle fibers. ${ }^{26}$

The increased extracellular potassium and increased intracellular sodium both contribute to the stimulation of the sodium pump. The pump is not fully activated in the paralysis phase, however, because salbutamol administration is still capable of stimulating the pump and relieving the weakness.

Hyperkalemia may not be necessary for the activation of mutant channels and the development of a paralytic attack, because T-tubular potassium accumulation (e.g., during myotonic activity) could be sufficient to locally 


\section{Skeletal muscle $\mathrm{Na}^{+}$channel $\left(\mathrm{Na}_{v} 1.4\right)$}

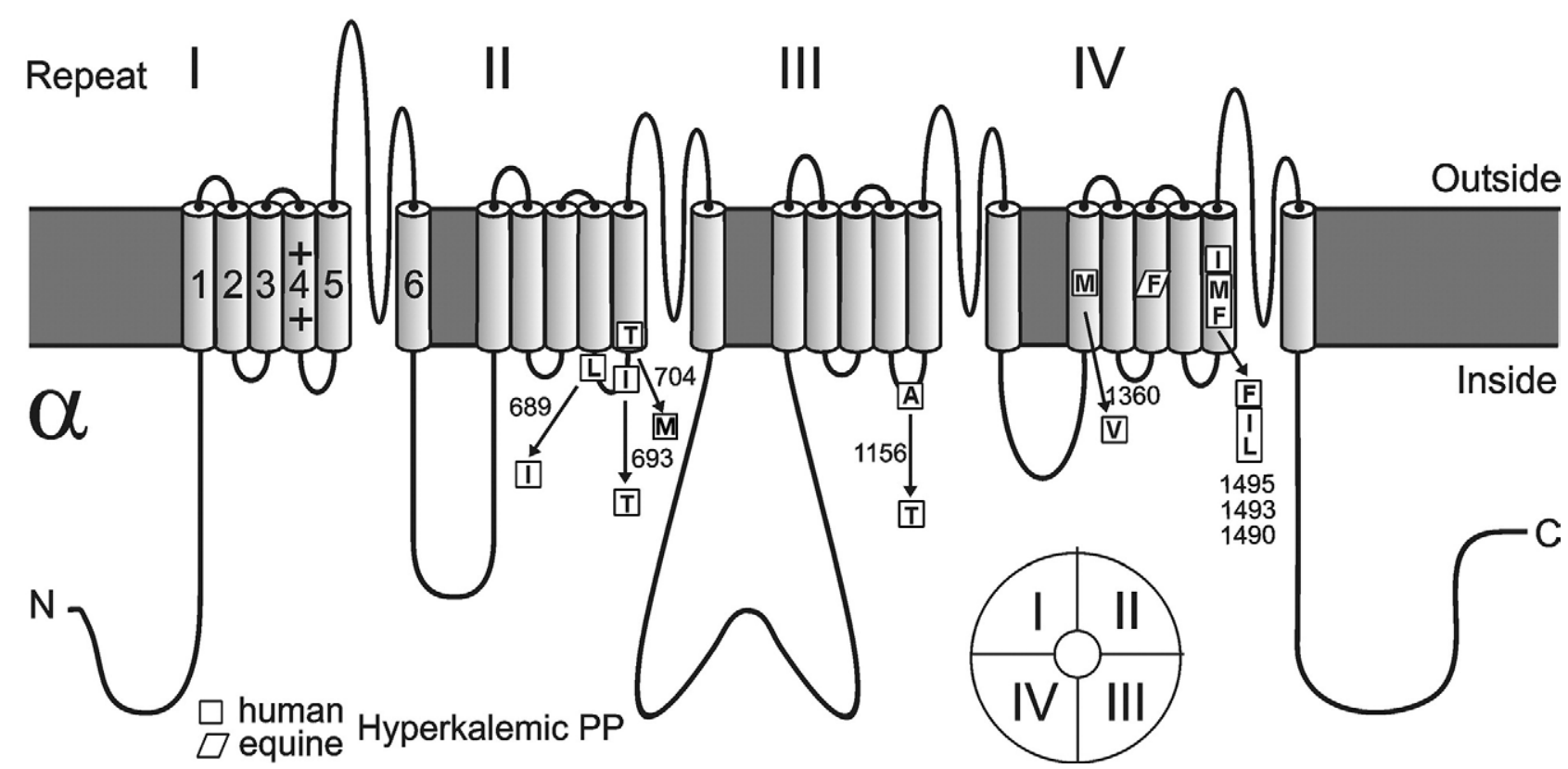

FIG. 2. Membrane topology model of the sodium channel of skeletal muscle, $\mathrm{Na}_{\mathrm{v}} 1.4$. The skeletal muscle $\alpha$-subunit, functioning as an ion-conducting channel, consists of four highly homologous domains (repeats I-IV) containing six transmembrane segments each (S1-S6). The S6 transmembrane segments and the S5-S6 loops form the ion selective pore, and the S4 segments contain positively charged residues conferring voltage dependence to the protein. The repeats are connected by intracellular loops; one of these, the III-IV linker, contains the supposed inactivation particle of the channel. When inserted in the membrane, the four repeats of the protein fold to generate a central pore (schematically indicated at the bottom right). The known mutations leading to hyperkalemic PP are indicated with the one-letter abbreviations of amino acids (boxed letters).

depolarize T-tubules, which would block the propagation of the action potential as well and thus cause normokalemic attacks.

The degree of membrane depolarization determines the clinical symptoms. The starting point for an attack is a slight membrane depolarization (e.g., by elevation of extracellular potassium due to ingestion or exercise), which opens the noninactivating mutant channels that generate repetitive muscle action potentials causing myotonia. Continued sodium channel opening enlarges the depolarization, so that the population of genetically normal sodium channels becomes inactivated and the muscle paralyzed. Although myotonia and paralysis are clinically the opposite, the pathomechanism is qualitatively the same.

\section{HYPERKALEMIC PP: IS THE INCOMPLETE SLOW INACTIVATION IMPORTANT FOR DISEASE SEVERITY?}

Slow inactivation determines channel availability over time period in the range of minutes, in contrast to fast inactivation, which is decisive in the range of seconds. Intact slow inactivation should terminate the influx of sodium ions into muscle fibers through channels with defective fast inactivation within several minutes. ${ }^{27} \mathrm{Sev}$ - eral studies ${ }^{28,29}$ showed that some but not all hyperkalemic PP-causing mutations reduce slow sodium channel inactivation and should thereby increase the permanent sodium influx into the muscle fibers, presumably generating long-lasting depolarization. In contrast, PC-causing mutations destabilize fast inactivation while slow channel inactivation is not affected. Therefore, sodium influx and membrane potential have been considered to be normal after a short delay. However, in vitro and in vivo studies have shown that increased sodium influx and membrane depolarization of cooled PC fibers persist for hours. ${ }^{24,25,30}$ This is in agreement with weakness lasting up to $8 \mathrm{~h}$ in PC patients, compared with that in hyperkalemic PP, which lasts from $30 \mathrm{~min}$ to $2 \mathrm{~h}$. The larger sodium influx and the more pronounced sustained depolarization in PC (by $40 \mathrm{mV}$ ) than in hyperkalemic PP (by $30 \mathrm{mV})^{24,25,30}$ may aggravate the T-tubular and myoplasmic disequilibrium and require more time for recovery.

In conclusion, a reduction of the slow inactivation is not needed as explanation for the paralytic episodes of hyperkalemic PP patients. In vivo study also clearly shows that $\mathrm{Na}$ influx, sustained depolarization, and muscle strength are highly correlated, and that cold environment should be considered as a typical reversible hy- 
$A$

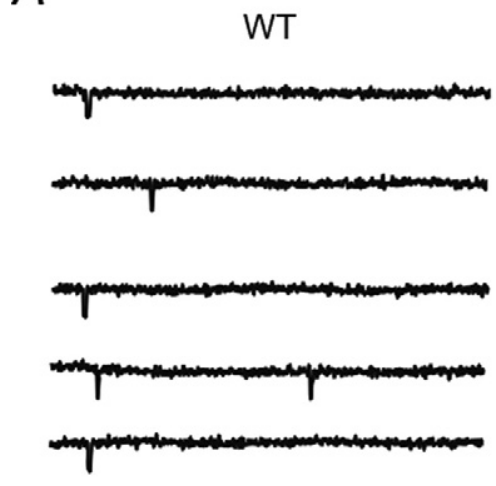

$B$

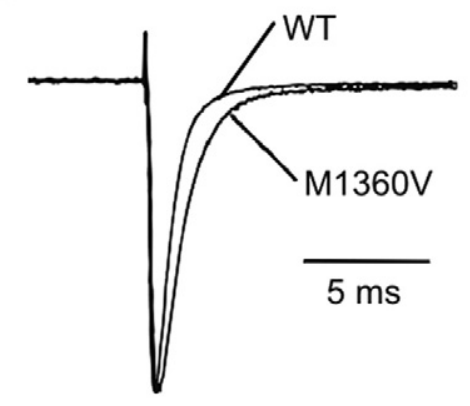

M1360V
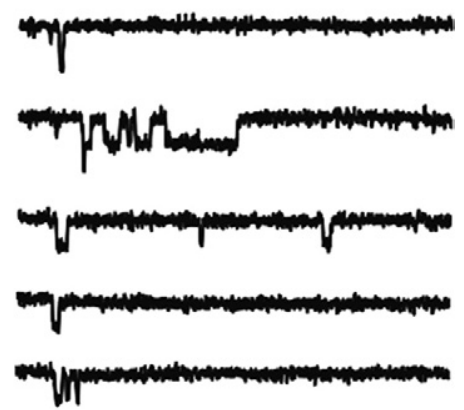

C

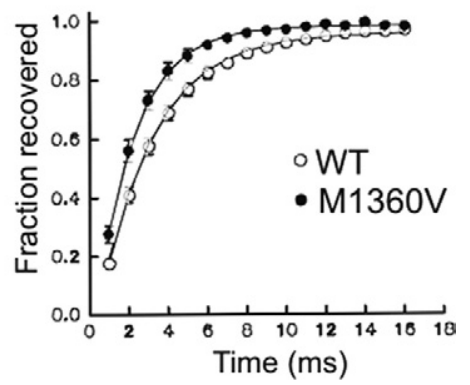

FIG. 3. Hyperkalemic periodic paralysis: Sodium current through mutant channels. (A) Current traces of single channel recordings for normal (wild type, WT) and mutant (M1360V) sodium channels. Channel openings were elicited by step depolarizations from a holding potential of $-100 \mathrm{mV}$ to $-30 \mathrm{mV}$. Reopenings were more frequent for mutant channels, thereby leading to a small permanent current. (B) Time course of inactivation of heterologously expressed sodium currents recorded in the whole-cell mode of the patch clamp technique. (C) Recovery from inactivation: sodium current amplitudes were measured at $-100 \mathrm{mV}$ following a variable latency after a $12 \mathrm{~ms}$ depolarization to $0 \mathrm{mV}$. Modified after Wagner et al., $1997 .{ }^{10}$

perkalemic PP trigger. ${ }^{25}$ In contrast to the cold-induced weakness in PC, hyperkalemic PP muscle regains strength after rewarming.

\section{HYPERKALEMIC PP AND PC: PHENOTYPIC OVERLAP}

The cardinal symptom of PC is cold-induced muscle stiffness that increases with continued activity (i.e., paradoxical myotonia). On intensive exercise and cooling, the stiffness may give way to paralysis. A characteristic clinical symptom is the inability to reopen the eyes after several forceful closures in rapid succession. Some, but not all, PC patients also have attacks of generalized hyperkalemic PP. This variability has led to a debate on whether hyperkalemic PP and PC are features of the same disease or different nosologic entities. In 1987, the debate was resolved by in vitro electrophysiological studies which established that both hyperkalemic PP and PC are associated with a noninactivating, tetrodotoxinsensitive sodium channel, ${ }^{24,30}$ conclusions that were confirmed by the finding that both types result from gain-of-function mutations of the gene encoding this channel.

Subsequent studies showed that PC families with typ- ical R1448 substitutions also have typical attacks of generalized hyperkalemic PP. In a Japanese family, the mutation M1370V resulted in PC in one family member and in hyperkalemic PP in others. ${ }^{20}$ In typical hyperkalemic PP mutations such as T704M and M1592V, paramyotonic signs have been reported in single families. ${ }^{31-33}$ The reason for the clinical variability is unknown.

Despite the striking overlap between hyperkalemic PP and PC, the therapy to be applied to a given patient is so different that the two allelic diseases should be considered as two clinical entities. Mexiletine had no effect on the spontaneous attacks of hyperkalemic weakness in PC patients with a R1448C PC mutation, whereas thiazide diuretics do not improve the paramyotonic features. ${ }^{34}$

\section{HYPERKALEMIC PP: PROVOCATIVE TESTS AND HISTOLOGY UNNECESSARY}

In the past, provocative tests were conducted for diagnostic reasons. Because such tests harbor the risk of inducing a severe attack, they had to be performed by an experienced physician and a standby anesthesiologist, and the serum potassium and glucose levels and the EKG had to be closely monitored. Nowadays, provocative tests should be restricted to patients in whom molecular 


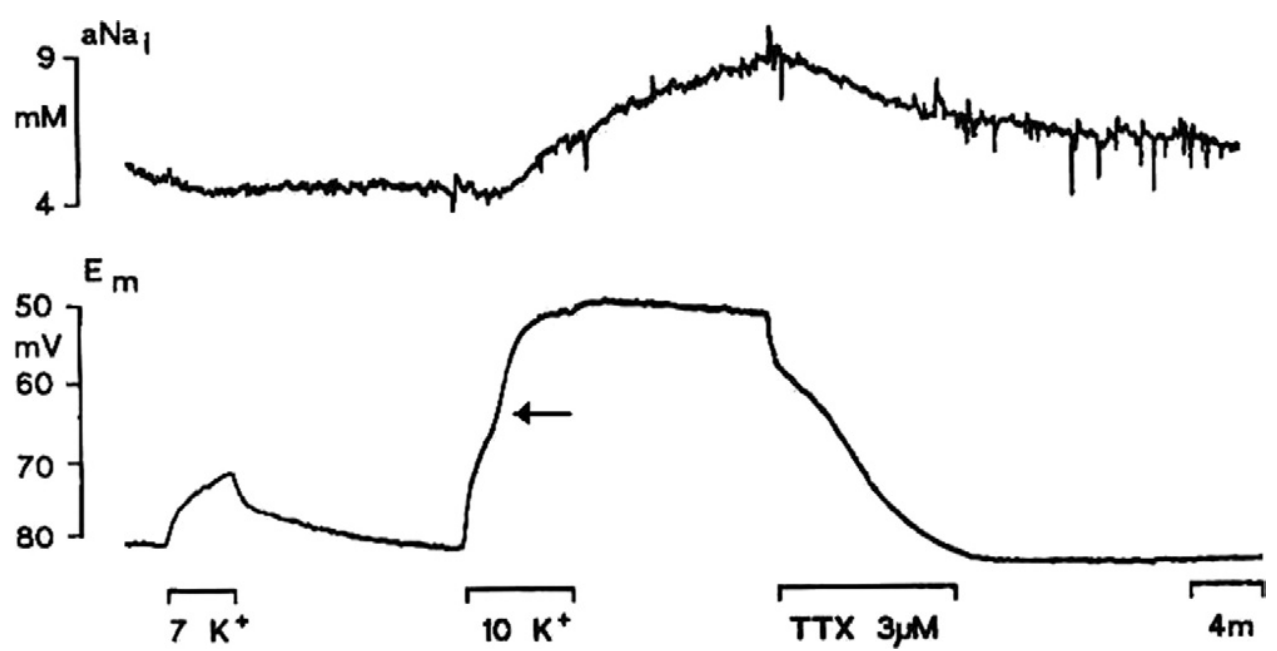

FIG. 4. Hyperkalemic periodic paralysis: Sodium influx in a muscle fiber as measured in vitro. The intracellular sodium activity aNa (upper panel) and the resting membrane potential $\mathrm{E}_{\mathrm{m}}$ (lower panel) from an excised muscle fiber from a hyperkalemic PP patient are shown. $\left[\mathrm{K}^{+}\right]_{\mathrm{o}}$ is $3.5 \mathrm{mmol} / \mathrm{L}(\mathrm{mM})$ unless indicated otherwise. In the presence of $10 \mathrm{mmol} / \mathrm{L}\left[\mathrm{K}^{+}\right]_{\mathrm{o}}$, the fiber shows a second phase of an abnormally large depolarization (arrow) and, associated with it, an increase in $\mathrm{aNa}_{\mathrm{i}}$. Blockade of the sodium channels by TTX speeds the reversal of $\mathrm{aNa}_{\mathrm{i}}$ and $\mathrm{E}_{\mathrm{m}}$. Modified after Lehmann-Horn et al., $1987 .{ }^{24}$

genetics fails to identify the mutation underlying the disease.

Muscle biopsy will not yield disease-specific results. The findings include proliferation, dilation, and degeneration of components of the T-tubular system and the sarcoplasmic reticulum, leading to the formation of vacuoles, ${ }^{35}$ as in a vacuolar myopathy. A muscle biopsy should therefore be performed only in patients with atypical features, or for the examination of the vacuolar myopathy or progressive muscle degeneration.

\section{HYPERKALEMIC PP: POTENTIAL LIFE-THREATENING COMPLICATIONS DURING GENERAL ANESTHESIA}

Opioids or depolarizing agents such as potassium, anticholinesterases, and succinylcholine can aggravate a myotonic reaction and induce masseter spasms and stiffness of respiratory muscles. ${ }^{36}$ Intubation and mechanical ventilation may be impaired. Also, alterations of serum osmolarity, $\mathrm{pH}$, and hypothermia-induced muscle shivering and mechanical stimuli can exacerbate the myotonic reaction. An induction sequence incorporating inhalation of oxygen, cricoid pressure, thiamylal or thiopental, and two times the $\mathrm{ED}_{95}$ dose of an intermediate- or short-action nondepolarizing muscle relaxant, followed by intubation, would be a reasonable approach to securing the airway in myotonic patients. ${ }^{37}$

Alternatively, inhalational induction may also be a possibility for hyperkalemic paralysis, and is well tolerated in the elective patient. Patients may develop respiratory distress in the recovery room, resulting from weakness of respiratory muscles in addition to generalized weakness lasting for hours. The weakness is aggra- vated by drugs that depress respiration and by the hypothermia induced by anesthesia. Preventive therapy before surgery (maintaining a normal body temperature, keep-
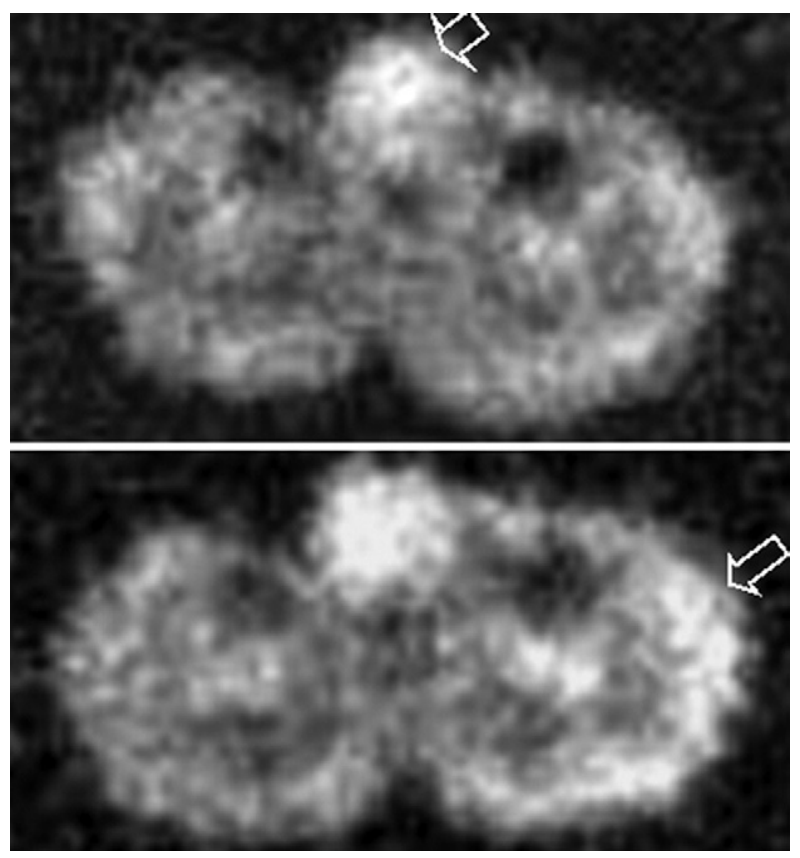

FIG. 5. Cold-induced increase in muscular ${ }^{23} \mathrm{Na}$ content in a hyperkalemic PP patient. Shown are cross sections of the lower legs of a 17-year-old male patient before (upper panel) and after (lower panel) cooling of the left lower leg. The $0.3 \% \mathrm{NaCl}$ solution reference phantom is positioned between both lower legs (upper panel, arrow). The ${ }^{23} \mathrm{Na} \mathrm{MRI}$ images after provocation reveal an increase in signal intensity in the cooled lower leg by $12 \%$ (Iower panel, arrow). The hyperkalemic PP patient developed a paresis for foot plantar flexion and dorsiflexion directly after cooling, with a Medical Research Council MRC score of 4, which remained unchanged at follow-up 30 min later. Modified after Weber et al., $2006 .^{25}$ 
ing serum potassium at a low level, and avoiding hypoglycemia) will help to prevent such attacks. ${ }^{38}$

\section{HYPERKALEMIC PP: A RATIONALE FOR THE THERAPY}

In hyperkalemic PP, physiological membrane depolarization can be prevented by avoiding potassium-retaining medications, potassium-rich foods, fasting, strenuous work, and exposure to cold. At the beginning of a paralytic attack, the serum potassium level can be reduced by stimulation of the sodium-potassium pump-for example, by continuous mild exercise or ingestion of carbohydrates or inhalation of salbutamol. Intravenous calcium gluconate may terminate attacks in some patients. It is often advisable to stabilize serum potassium levels at the low normal range with thiazide diuretics. Carbonic anhydrase inhibitors are the second choice and may be effective via myoplasmic acidification (for a review, see the chapter by Lehmann-Horn et al., 2004 ${ }^{1}$ ) or activation of potassium channels, which may support fiber repolarization. ${ }^{39}$ This has been shown in a model of hypokalemic PP, but may also apply to hyperkalemic PP.

\section{ANDERSEN-TAWIL SYNDROME: DIFFERENTIAL DIAGNOSIS OF HYPERKALEMIC PP}

Andersen's syndrome is characterized by the highly variable clinical triad of dyskalemic PP, ventricular ectopy, and potential dysmorphic features. ${ }^{40}$ Patients may experience a life-threatening ventricular arrhythmia independent of the PP, and long QT syndrome is the primary cardiac manifestation. ${ }^{41,42}$ Mutations of the Kir2.1 potassium channel, an inward rectifier expressed in skeletal and cardiac muscle, are causative of the disorder. ${ }^{43}$ Although the paralytic attacks can be hyper-, normo- or hypokalemic (and, accordingly, the response to oral $\mathrm{K}^{+}$ is considered to be unpredictable), certain mutations such as $\mathrm{G} 215 \mathrm{D}$ and $\mathrm{R} 218 \mathrm{~W}$ seem to be associated rather with hyperkalemia than with hypokalemia. ${ }^{44,45}$ More studies are needed to substantiate these preliminary results. However, it has been well established that the patients who were hyperkalemic during an attack did not reveal any clinical or electromyographic signs of myotonia.

\section{HYPERKALEMIC PP: CONCLUSIONS FROM RECENT GENETIC STUDIES}

In 2001, an R83H substitution in a $\mathrm{K}^{+}$channel $\beta$-subunit, MiRP2, was suggested to cause hyperkalemic PP in one and hypokalemic PP in another of 100 such patients because it showed a loss of function in vitro and was not found in 120 unaffected controls. ${ }^{46}$ In subsequent studies, the substitution was identified in 1 of 104 and 1 of
138 patients, but also in 8 of 506 and 3 of 321 controls. $^{47,48}$ Taken together, the substitution is present in $1.17 \%$ of patients and in $1.16 \%$ of healthy controls, which does not support disease causality and shows that the common laboratory practice to exclude a novel mutation in approximately 100 healthy controls is insufficient. The number of controls required to prove disease causality of a putative mutation can be calculated by a simple equation. ${ }^{47}$

Even though the difference between defining a putative mutation as being truly disease-causing or as being a functional polymorphism may seem only marginal on a scientific level, this difference in definition has drastic consequences for an affected carrier whose diagnosis is made or confirmed by the finding and who is being medically treated. This problem will increasingly need to be addressed in future studies, as the number of known mutations and putatively associated phenotypes continues to increase.

\section{HYPERKALEMIC PP: NATURALLY OCCURRING AND EXPERIMENTAL MODELS}

A condition equivalent to human hyperkalemic PP has been identified in the American Quarter Horse, a common breed of race horses in the United States. Its incidence is highest of all known hereditary horse diseases. The symptoms are similar, but the condition seems to be more serious than in humans, in that several affected horses have died during attacks. The hyperexcitability of all muscles of the affected Quarter Horses causes hypertrophy, and it is the aesthetic appeal resulting in particular beauty that makes them show winners instead of race winners.

A mutation was identified in the equine muscle sodium channel gene, the homolog to SCN $4 A{ }^{49}$ All affected horses $(4.4 \%$ of the quarter horses in the United States) trace to the same sire, Impressive, as first, second or third generation descendants. Thus, the disorder is an ideal model for the study of the cellular and physiological factors dictating the onset and severity of attacks and the relationship between exercise, serum potassium levels, catecholamines, and other factors influencing muscle metabolism.

A decrease in serum sodium is followed by an increase in potassium and entrance of water into the fibers, causing hemoconcentration and further increase in serum potassium. ${ }^{50}$ The study of the hyperkalemic horse also revealed the first correlation of mutant relative to normal mRNA level as a likely determinant of clinical severity in a dominantly inherited disease. ${ }^{51}$ Accordingly, homozygous animals had laryngeal and pharyngeal dysfunction during exercise, whereas heterozygous animals did not, even though their weakness and myotonia were comparable. ${ }^{52}$ As in human patients, the primary physi- 
ological defect in mutant sodium channels was an impairment of inactivation. This defect was manifest as bursts of persistent activity during which the channel closed and reopened throughout a maintained depolarization. ${ }^{53,54}$

A peptide toxin (ATX II) from a sea anemone, Anemonia sulcata, selectively disrupts the inactivation mechanism of sodium channels in a way that mimics sodium channel mutations causing myotonia. The toxin causes reopenings of the channels similar to those observed in hyperkalemic PP. ${ }^{55} \mathrm{~A}$ model provided a possible mechanism for how this small persistent sodium current leads to repetitive firing, why the integrity of the T-tubular system is required to produce myotonia, and why paralysis will occur when a slightly larger proportion of channels fails to inactivate. ${ }^{56}$

\section{HYPERKALEMIC PP: OPEN QUESTIONS}

Unsolved problems include the occurrence of vacuolar myopathy and progressive fatty muscle degeneration. T-tubular disturbances of ion concentration may lead to swelling and proliferation of these structures and thus cause the vacuolar myopathy (for a review, see the chapter by Lehmann-Horn et al., 2004 ${ }^{1}$ ). The fatty degeneration, however, which occurs particularly in T704M carriers, may not result from the same mechanism. As shown in Figure 1, even myotonia that usually results in muscle hypertrophy cannot prevent the muscle degeneration that goes along with atrophy. Both types of structural changes need to be studied more extensively.

Although mexiletine seems not to be of benefit in hyperkalemic PP, more emphasis is needed on testing whether the pathologically increased sodium influx through the mutant channels (particularly T704M) can be blocked by appropriate substances. Hence, a pharmacogenetic strategy for mutation-specific treatment is needed. ${ }^{57,58}$ Another unclear issue is whether the cardiac arrests reported in the premolecular era ${ }^{5,6,7}$ occurred in patients with hyperkalemic PP or Andersen-Tawil syndrome. Last but not least, the question of why certain mutations cause hyperkalemic PP in some families or family members and PC in others has not yet been clarified.

\section{REFERENCES}

1. Lehmann-Horn F, Rüdel R, Jurkat-Rott K. Nondystrophic myotonias and periodic paralyses. In: Engel AG, Franzini-Armstrong C, editors. Myology: basic and clinical. Vol. 2. 3rd ed. New York: McGraw-Hill; 2004:1257-1300.

2. Ricker K, Camacho LM, Grafe P, et al. Adynamia episodica hereditaria: what causes the weakness? Muscle Nerve 1989;12:883891.

3. Bradley WG, Taylor R, Rice DR, et al. Progressive myopathy in hyperkalemic periodic paralysis. Arch Neurol 1990;47:1013-1017.

4. Lehmann-Horn F, Rüdel R, Ricker K. Non-dystrophic myotonias and periodic paralyses. A European Neuromuscular Center Work- shop held 4-6 October 1992, Ulm, Germany. Neuromuscul Disord 1993;3:161-168.

5. Gould RJ, Steeg CN, Eastwood AB, et al. Potentially fatal cardiac dysrhythmia and hyperkalemic periodic paralysis. Neurology 1985;35:1208-1212.

6. Lisak RP, Lebeau J, Tucker SH, et al. Hyperkalemic periodic paralysis and cardiac arrhythmia. Neurology 1972;22:810-815.

7. Yoshimura T, Kaneuji M, Okuno T, et al. Periodic paralysis with cardiac arrhythmia. Eur J Pediatr 1983;140:338-343.

8. Jurkat-Rott K, Lehmann-Horn F. Hyperkalemic periodic paralysis type I. Gene Reviews at GeneTests 2005; Available at: http:// www.genetests.org, Medical Genetics Information Resource [database online]. Funded by NIH. Copyright, University of Washington, Seattle.

9. McClatchey AI, McKenna-Yasek D, Cros D, et al. Novel mutations in families with unusual and variable disorders of the skeletal muscle sodium channel. Nat Genet 1992;2:148-152.

10. Wagner S, Lerche H, Mitrovic N, et al. A novel sodium channel mutation causing a hyperkalemic paralytic and paramyotonic syndrome with variable clinical expressivity. Neurology 1997;49: $1018-1025$.

11. Meyers KR, Gilden DH, Rinaldi CF, et al. Periodic muscle weakness, normokalemia, and tubular aggregates. Neurology 1972;22: 269-279.

12. Poskanzer DC, Kerr DNS. A third type of periodic paralysis with normokalemia and favorable response to sodium chloride. Am J Med 1961;31:328-342.

13. Chinnery PF, Walls TJ, Hanna MG, et al. Normokalemic periodic paralysis revisited: does it exist? Ann Neurol 2002;52:251-252.

14. Vicart S, Sternberg D, Fournier E, et al. New mutations of SCN4A cause a potassium-sensitive normokalemic periodic paralysis. Neurology 2004;63:2120-2127.

15. Fontaine B, Khurana TS, Hoffman EP, et al. Hyperkalemic periodic paralysis and the adult muscle sodium channel alpha-subunit gene. Science 1990;250:1000-1002.

16. Rojas CV, Wang J, Schwartz L, et al. A Met-to-Val mutation in the skeletal muscle sodium channel $\alpha$-subunit in hyperkalemic periodic paralysis. Nature 1991;354:387-389.

17. Bendahhou S, Cummins TR, Kula RW, Fu YH, Ptacek LJ. Impairment of slow inactivation as a common mechanism for periodic paralysis in DIIS4-S5. Neurology 2002;58:1266-1272.

18. Plassart E, Eymard B, Maurs L, et al. Paramyotonia congenita: genotype to phenotype correlations in two families and report of a new mutation in the sodium channel gene. J Neurol Sci 1996;142: 126-133.

19. Ptacek LJ, George AL Jr, Griggs RC, et al. Identification of a mutation in the gene causing hyperkalemic periodic paralysis. Cell 1991;67:1021-1027.

20. Okuda S, Kanda F, Nishimoto K, et al. Hyperkalemic periodic paralysis and paramyotonia congenita: a novel sodium channel mutation. J Neurol 2001;248:1003-1004.

21. Bendahhou S, Cummins TR, Tawil R, et al. Activation and inactivation of the voltage-gated sodium channel: role of segment S5 revealed by a novel hyperkalaemic periodic paralysis mutation. J Neurosci 1999;19:4762-4771.

22. Bendahhou S, Cummins TR, Hahn AF, et al. A double mutation in families with periodic paralysis defines new aspects of sodium channel slow inactivation. J Clin Invest 2000:106:431-438.

23. Lehmann-Horn F, Iaizzo PA, Hatt $\mathrm{H}$, et al. Altered gating and conductance of $\mathrm{Na}^{+}$channels in hyperkalemic periodic paralysis. Pflugers Arch 1991;418:297-299.

24. Lehmann-Horn F, Kuther G, Ricker K, et al. Adynamia episodica hereditaria with myotonia: a non-inactivating sodium current and the effect of extracellular pH. Muscle Nerve 1987;10:363-374.

25. Weber M-A, Nielles-Vallespin S, Essig $\mathrm{M}$, et al. Muscle $\mathrm{Na}^{+}$ channelopathies: MRI detects intracellular ${ }^{23} \mathrm{Na}$ accumulation during episodic weakness. Neurology 2006;67:1151-1158.

26. Lehmann-Horn F, Jurkat-Rott K. Voltage-gated ion channels and hereditary disease. Physiol Rev 1999;79:1317-1371.

27. Ruff RL. Slow $\mathrm{Na}^{+}$channel inactivation must be disrupted to evoke prolonged depolarization-induced paralysis. Biophys J 1994;66:542. 
28. Hayward LJ, Brown RH Jr, Cannon SC. Slow inactivation differs among mutant $\mathrm{Na}$ channels associated with myotonia and periodic paralysis. Biophys J 1997;72:1204-1219.

29. Ruff RL, Cannon SC. Defective slow inactivation of sodium channels contributes to familial periodic paralysis. Neurology 2000;54: $2190-2192$.

30. Lehmann-Horn F, Rüdel R, Ricker K. Membrane defects in paramyotonia congenita (Eulenburg). Muscle Nerve 1987;10:633641.

31. Kelly P, Yang WS, Costigan D, et al. Paramyotonia congenita and hyperkalemic periodic paralysis associated with a Met $1592 \mathrm{Val}$ substitution in the skeletal muscle sodium channel $\alpha$ subunit: a large kindred with a novel phenotype. Neuromuscul Disord 1997; $7: 105-111$.

32. Kim J, Hahn Y, Sohn EH, et al. Phenotypic variation of a Thr704Met mutation in skeletal sodium channel gene in a family with paralysis periodica paramyotonica. J Neurol Neurosurg Psychiatry 2001;70:618-623.

33. Brancati F, Valente EM, Davies NP, et al. Severe infantile hyperkalaemic periodic paralysis and paramyotonia congenita: broadening the clinical spectrum associated with the T704M mutation in SCN4A. J Neurol Neurosurg Psychiatry 2003;74:13391341.

34. Ricker K, Böhlen R, Rohkamm R. Different effectiveness of tocainide and hydrochlorothiazide in paramyotonia congenita with hyperkalemic episodic paralysis. Neurology 1983;33:1615-1618.

35. Jurkat-Rott K, Müller-Höcker J, Pongratz D, et al. Diseases associated with ion channel and ion transporter defects: dyskalemic episodic weakness. In: Karpati G, editor. Structural and molecular basis of skeletal muscle diseases. Basel: ISN Neuropath Press; 2002:95-98.

36. Russell SH, Hirsch NP. Anaesthesia and myotonia. Br J Anaesth 1994;72:210-216.

37. Neuman GG, Kopman AF. Dyskalemic periodic paralysis and myotonia. Anesth Analg 1993;76:426-428.

38. Klingler W, Lehmann-Horn F, Jurkat-Rott K. Complications of anaesthesia in neuromuscular disorders. Neuromuscul Disord 2005;15:195-206.

39. Tricarico D, Barbieri M, Mele A, Carbonara G, Conte Camerino D. Carbonic anhydrase inhibitors are specific openers of skeletal muscle BK channel of $\mathrm{K}^{+}$-deficient rats. FASEB J 2004;18:760-761.

40. Tawil R, Ptacek LJ, Pavlakis SG, et al. Andersen's syndrome: potassium-sensitive periodic paralysis, ventricular ectopy, and dysmorphic features. Ann Neurol 1994;35:326-330.

41. Andelfinger G, Tapper AR, Welch RC, et al. KCNJ2 mutation results in Andersen syndrome with sex-specific cardiac and skeletal muscle phenotypes. Am J Hum Genet 2002;71:663-668.

42. Tristani-Firouzi M, Jensen JL, Donaldson MR, et al. Functional and clinical characterization of $K C N J 2$ mutations associated with LQT7 (Andersen syndrome). J Clin Invest 2002;110:381-388.

43. Plaster NM, Tawil R, Tristani-Firouzi M, et al. Mutations in Kir2.1 cause the developmental and episodic electrical phenotypes of Andersen's syndrome. Cell 2001;105:511-519.
44. Hosaka Y, Hanawa H, Washizuka T, et al. Function, subcellular localization and assembly of a novel mutation of KCNJ2 in Andersen's syndrome. J Mol Cell Cardiol 2003;35:409-415.

45. Zhang L, Benson DW, Tristani-Firouzi M, et al. Electrocardiographic features in Andersen-Tawil syndrome patients with KCNJ2 mutations: characteristic T-U-wave patterns predict the KCNJ2 genotype. Circulation 2005;111:2720-2726.

46. Abbott GW, Butler MH, Bendahhou S, et al. MiRP2 forms potassium channels in skeletal muscle with Kv3.4 and is associated with periodic paralysis. Cell 2001;104:217-231.

47. Jurkat-Rott K, Lehmann-Horn F. Periodic paralysis mutation MiRP2-R83H in controls: interpretations and general recommendation. Neurology 2004;62:1012-1015.

48. Sternberg D, Tabti N, Fournier E, et al. Lack of association of the potassium channel-associated peptide MiRP2-R83H variant with periodic paralysis. Neurology 2003;61:857-859.

49. Rudolph JA, Spier SJ, Byrns G, Rojas CV, Bernoco D, Hoffman EP. Periodic paralysis in quarter horses: a sodium channel mutation disseminated by selective breeding. Nat Genet 1992;2:144147.

50. Spier SJ, Carlson GP, Holliday TA, Cardinet GH 3rd, Pickar JG. Hyperkalemic periodic paralysis in horses. J Am Vet Med Assoc 1990;197:1009-1017.

51. Zhou J, Spier SJ, Beech J, Hoffman EP. Pathophysiology of sodium channelopathies: correlation of normal/mutant mRNA ratios with clinical phenotype in dominantly inherited periodic paralysis. Hum Mol Genet 1994;3:1599-1603.

52. Carr EA, Spier SJ, Kortz GD, Hoffman EP. Laryngeal and pharyngeal dysfunction in horses homozygous for hyperkalemic periodic paralysis. J Am Vet Med Assoc 1996;209:798-803.

53. Cannon SC, Hayward LJ, Beech J, Brown RH Jr. Sodium channel inactivation is impaired in equine hyperkalemic periodic paralysis. J Neurophysiol 1995;73:1892-1899.

54. Hanna WJ, Tsushima RG, Sah R, McCutcheon LJ, Marban E, Backx PH. The equine periodic paralysis $\mathrm{Na}^{+}$channel mutation alters molecular transitions between the open and inactivated states. J Physiol 1996;497:349-364.

55. Cannon SC, Corey DP. Loss of $\mathrm{Na}^{+}$channel inactivation by anemone toxin (ATX II) mimics the myotonic state in hyperkalaemic periodic paralysis. J Physiol 1993;466:501-520.

56. Cannon SC, Brown RH Jr, Corey DP. Theoretical reconstruction of myotonia and paralysis caused by incomplete inactivation of sodium channels. Biophys J 1993;65:270-288.

57. Jurkat-Rott K, Lehmann-Horn F. Muscle channelopathies and critical points in functional and genetic studies. J Clin Invest 2005; 115:2000-2009.

58. Desaphy JF, De Luca A, Tortorella P, De Vito D, George AL Jr, Conte Camerino D. Gating of myotonic Na channel mutants defines the response to mexiletine and a potent derivative. Neurology 2001;57:1849-1857. 